\title{
INFLUENCIA DE ALGUNAS VARIABLES MÉDICAS Y PSICOSOCIALES EN LA RECUPERACIÓN PSICOLÓGICA DE LOS TRASPLANTADOS. FUTURAS LÍNEAS DE INTERVENCIÓN PSICOLÓGICA
}

\author{
M. ÁNGELES PÉREZ ${ }^{1}$, AGUSTÍN MARTIIN ${ }^{1}$, ANA GALLEGO ${ }^{2}$ y JOSÉ L. SANTAMARÍ ${ }^{2}$ \\ ${ }^{1}$ Facultad de Psicología de Sevilla \\ ${ }^{2}$ Hospital Universitario "Virgen del Rocío" de Sevilla
}

(Recibido el 8 de octubre de 1999)

En la presente investigación analizamos la influencia que ejerce en la recuperación psicológica (ansiedad, depresión, tipo de pensamientos, autoconcepto físico y conductas de afrontamiento) de los trasplantados una serie de variables médicas (tipo de órgano trasplantado y hospitalización) y psicosociales (tiempo transcurrido desde el implante, expectativas hacia la enfermedad y tipo de relaciones familiares tras el trasplante). Para lograr estos objetivos empleamos una muestra constituida por 100 pacientes trasplantados: 59 de riñón, 23 de hígado y 18 de corazón. Todos completaron una encuesta psicosocial (datos sociodemográficos, médicos, etc.) y una batería de instrumentos psicológicos: cuestionario de ansiedad estado-rasgo (Spielberger, Gorsuch y Lushene, 1982), inventario de depresión de Beck (Beck, Brown, Eidelson, Steer y Riskind, 1987), escala de autoconcepto físico (Garanto, 1984) y cuestionario de conductas de afrontamiento (Muñoz, 1988). Tras analizar los resultados hallamos, por un lado, que el tipo de órgano trasplantado y el tiempo de hospitalización tras el trasplante no ejercen una influencia importante en la recuperación psicológica de los trasplantados y, por otro lado, que los desequilibrios psicológicos de estos pacientes aumentan cuando ingresan en el hospital debido a alguna complicación médica tras el implante cardíaco (trasplantados cardíacos), durante el primer año (0-12 meses) y a partir de los dos años ( $>24$ meses) de haber recibido el implante renal (trasplantados renales), si tienen unas expectativas negativas hacia su enfermedad y cuando sus relaciones familiares empeoran tras el trasplante. A partir de estos resultados, proponemos algunas estrategias de intervención psicológica con el objetivo de favorecer una adecuada evolución psicológica en los pacientes trasplantados.

Palabras clave: Trasplante de 6́rganos, evolución psicológica, hospitalización, expectativas hacia la enfermedad, relaciones familiares.

\section{Influences of some medical and psychosocial variables of the transplant patients' psychological recovery. Future lines of psychological intervention}

In the present investigation we analyze the influence that some medical (type of transplanted organ and hospitalization) and psychosocial variables (period from the implant, expectations toward the illness and type of family relationships from the transplant) exercises in the psychological recovery (anxiety, depression, type of thoughts, physical self-concept and coping behaviors) of the transplant patients. To achieve these objectives we use a sample composed of 100 transplant patients: 59 of kidney, 23 of liver and 18 of heart. All of them filled a psychosocial questionnaire (sociodemographics data, medicals data, etc) and a battery of psychological instruments: state-trait anxiety inventory (Spielberger, Gorsuch y Lushene, 1982), Beck's depression inventory (Beck, Brown, Eidelson, Steer y Riskind, 1987), physical self-concept scale (Garanto, 1984) and coping behaviors questionnaire (Muñoz, 1988). After

Agradecimientos: Los autores desean expresar su agradecimiento a la administrativa de la Unidad Sectorial de Coordinación de Trasplentes, a las Asociaciones de Trasplantados Renales, Hepáticos y Cardiacos de Sevilla y a todos los trasplantados por su participación en este estudio.
Correspondencia: M. Ángeles Pérez, Facultad de Psicología, Departamento de Psiquiatría, Personalidad, Evaluación y Tratamiento Psicológicos, Avenida de San Francisco Javier, s/n, 41005 Sevilla. Tfnos.: 954 556 939/ 954401 786. Fax: 954557807. 


\begin{abstract}
analyzing the results we find on one hand that the type of transplanted organ and the time of hospitalization after the transplant don't exercise an important influence in the psychological recovery of the transplant patients and, on the other hand that the psychological disorders of these patients increase when they enter in the hospital due to some medical complication after the heart implant (heart transplant patients), during first year (0-12 months) and from two years (>24 months) of having received the renal implant (kidney transplant patients), they have some negative expectations toward their illness and when their family relationships are worse after the transplant. From these results, we propose some strategies of psychological intervention in the order to favour an appropriate psychological evolution in the transplant patients.
\end{abstract}

Key words: Organ transplantation, psychological evolution, hospitalization, expectations toward the illness, family relationships.

\section{INTRODUCCIÓN}

El trasplante de órganos es una alternativa terapéutica para muchos pacientes que sufren insuficiencia renal, hepática, cardiaca, pulmonar, etc. Sin embargo, aunque la Medicina ha realizado avances espectaculares en este ámbito, desde la Psicología ha sido un tema olvidado. En este sentido, los escasos estudios existentes se centran en analizar la sintomatología psicológica que presentan los pacientes trasplantados en comparación con la población normal pero, la mayoría de ellos, olvidan la influencia que en dichos síntomas pueden ejercer variables como los episodios de rechazo, el tipo de órgano trasplantado, el apoyo de la familia y del equipo de trasplantes, etc.

En líneas generales, los trastornos psicológicos que suelen aparecer tras el trasplante de órganos podríamos resumirlos en los siguientes:

- Alteraciones del estado de ánimo: lo más frecuente es la depresión causada, en muchas ocasiones, por la aparición de complicaciones médicas o por el temor al rechazo y a una evolución desfavorable del trasplante (Menchón, González, Aymamí, Barjau y Serrano, 1997; Plutchik, Snyder, Drooker, Chodoff y Sheiner, 1998; Zipfel et al., 1998).

- Trastornos de ansiedad: algunos trasplantados padecen trastorno por estrés postraumático. Esta alteración se observa especialmente en los trasplantados cardíacos con antecedentes psiquiátricos, con escaso apoyo familiar y con estrategias de afrontamiento evitativas para manejar los problemas de salud (Dew, Roth, Schulberg y Simmons, 1996; Stukas et al., 1999).

- Disfunciones sexuales: los trasplantados suelen presentar alteraciones del deseo, de la excitación y del orgasmo, especialmente los cardíacos, ya que mucho de estos pacientes consideran que su corazón está debilitado y que no pueden realizar ningún tipo de esfuerzo, de ahí que teman la muerte durante el coito (Tabler y Frieson, 1990). En concreto, de un 22 a un $43 \%$ de los hombres trasplantados sufren trastornos de la erección (House y Thompson, 1988).

- Alteraciones neurológicas: las más importantes según un estudio realizado por López, Estol y Colina (1992), con una muestra de 185 trasplantados hepáticos son las siguientes: alteraciones del estado mental, crisis epilépticas y déficit de la psicomotricidad.

- Alteraciones de la imagen corporal: al principio, el nuevo órgano es un cuerpo extraño que ha de integrarse en la nueva imagen somática que el receptor tiene de sí mismo. A veces este proceso fracasa y los pacientes se sienten confeccionados con trozos de 
cadáveres, lo que repercute negativamente en su estado anímico (Van Der Velde, 1985).

- Problemas de identificación con el donante y sus familiares: tras el trasplante, muchos pacientes desean conocer las características del donante: sexo, edad, raza, etc. y, en algunas ocasiones, se produce una identificación del receptor con el donante y se intenta ser como éste, para que parcialmente siga vivo. Se han descrito casos de amistad entre el trasplantado y la familia del fallecido, incluso hay recogidos en la literatura casos de matrimonios con la viuda del donante (Iruela, Merino y Lombardía, 1995).

Cuando aparecen todas estas complicaciones psicológicas, los pacientes suelen culpar al personal sanitario, no obstante, hay que tener en cuenta que todas ellas disminuyen a medida que el paciente mejora físicamente (House y Thompson, 1988). A pesar de todas estas repercusiones psicológicas, la cantidad y calidad de vida de un trasplantado es muy superior a la que tenía antes de recibir el implante. Esto se manifiesta por el hecho de que tienen menos problemas en su movilidad física, autocuidado y relaciones sociales y personales y en que, además, la hospitalización es menos frecuente (Aranzábal y Magaz, 1993; Evans, 1985; Gudex, 1995; Simmons, Kamstra y Thompson, 1981).

Dadas las repercusiones psicologicas del trasplante de órganos y la escasez de estudios sobre este tema, en la presente investigación analizamos la influencia que ejercen algunas variables médicas y psicosociales en la recuperación psicológica de los trasplantados. Concretamente, cómo la ansiedad (estado y rasgo), la depresión, los pensamientos (ansiosos y depresivos), el autoconcepto (autoconcepto físico, autoestima física y autocomportamiento físico) y las conductas de afrontamiento (directo, pasivo y escape-evitación) sufren algunos cambios debido a la influencia de variables médicas (tipo de órgano trasplantado y hospitalización) y psicosociales (tiempo transcurrido desde el implante, expectativas hacia la enfermedad y tipo de relaciones familiares tras el trasplante). Este objetivo general, podríamos desglosarlo en los siguientes objetivos específicos:

\section{A) Variables médicas:}

- Tipo de órgano trasplantado: analizar las diferencias psicológicas entre distintos grupos de trasplantados: renales, hepáticos y cardíacos.

- Hospitalización: analizar si existen diferencias psicológicas en función, por un lado, del tiempo de hospitalización del paciente tras la implantación del injerto y, por otro lado, de los ingresos en el hospital debidos a complicaciones médicas tras el trasplante.

B) Variables psicosociales:

- Tiempo transcurrido desde el implante: analizar si existen diferencias psicológicas en función del tiempo transcurrido (un año, entre un año y dos años y más de dos años) desde que el paciente fue trasplantado.

- Expectativas hacia la enfermedad: analizar si existen diferencias psicológicas entre los trasplantados según sus diferentes expectativas (más o menos positivas) hacia su enfermedad y sus actitudes ante el hecho de estar enfermo.

- Tipo de relaciones familiares tras el trasplante: analizar si existen diferencias psicológicas entre los trasplantados en función de cómo sean las relaciones entre los miembros de la familia a raíz del trasplante: mejores, peores o las mismas que antes de ser trasplantado. 
C) En función de los resultados, proponer futuras líneas de intervención psicológica que favorezcan una adecuada evolución psicológica de los pacientes que han sido sometidos a un trasplante de órganos.

\section{MÉTODO}

\section{Muestra}

Para la realización del presente estudio, hemos utilizado un grupo constituido por 100 pacientes trasplantados con una edad media de 47 años. El $64 \%$ eran hombres y el $36 \%$ mujeres. La mayoría $(77,8 \%)$ estaban casados o vivían en pareja y su nivel educativo era mediobajo (el $40 \%$ había realizado sólo los estudios primarios completos y el $29 \%$ sabía leer y escribir pero no tenía estudios).

Estos pacientes habían sido sometidos a diferentes tipos de trasplante de 6rganos: riñón (59\%), hígado (23\%) y corazón (18\%). Tras el implante, los pacientes permanecieron ingresados en el hospital aproximadamente 25 días y sólo un $29 \%$ tuvo que reingresar debido a alguna complicación médica tras el trasplante. La mayoría seguía fielmente las pautas de tratamiento aconsejadas por el médico (98\%) y asistía a las revisiones médicas para su trasplante ( $98 \%$ ). Además, estaban muy satisfechos tanto con las actuaciones médicas e intervención quirúrgica ( $98 \%$ ) como con la información recibida sobre todo el proceso de trasplante $(87,7 \%)$.

\section{Selección de la muestra}

Tras obtener el informe favorable del «Comité Ético de Investigación Clínica» del Hospital Universitario "Virgen del Rocío» para poder llevar a cabo este estudio, elaboramos con la ayuda de la administrativa de la Oficina de la Unidad Sectorial de Coordinación de Trasplantes, un listado de los trasplantadosk renales, hepáticos y cardíacos de Sevilla y provincia durante el período enero (1995)-octubre (1998). Tras eliminar los pacientes que habían fallecido, seleccionamos 243 trasplantados, cuya distribución en función del órgano implantado y de la fecha del trasplante podemos apreciarla en la Tabla 1.

\section{Procedimiento}

Enviamos al domicilio personal de los 243 trasplantados un sobre con la siguiente documentación: (1) una carta firmada por el Coordinador Sectorial de Trasplantes explicándole los objetivos del presente estudio y solicitándole su colaboración voluntaria, (2) una encuesta psicosocial y una batería de instrumentos psicológicos, y (3) un sobre franqueado y con la dirección de la Unidad

Tabla 1. Número de pacientes trasplantados no fallecidos. Sevilla y provincia (enero 1995/octubre 1998)

\begin{tabular}{lcccc}
\hline & \multicolumn{3}{c}{ Tipo de trasplante } \\
\cline { 2 - 4 } $\begin{array}{c}\text { Fecha del } \\
\text { Trasplante }\end{array}$ & Riñón & Hígado & Corazón & Total \\
\hline 1995 & 41 & 15 & 7 & 63 \\
1996 & 39 & 11 & 5 & 55 \\
1997 & 36 & 15 & 8 & 59 \\
1998 & 47 & 14 & 5 & 66 \\
Total & 163 & 55 & 25 & 243 \\
\hline
\end{tabular}


Sectorial de Coordinación de Trasplantes; lugar donde tenían que enviar la documentación tras completar el protocolo de evaluación.

Los sobres fueron enviados en las siguientes fechas: 22-12-98 (pacientes renales que habían sido trasplantados en el año 1995 y todos los trasplantados hepáticos), 08-01-1999 (trasplantados cardíacos) y 14-01-1999 (resto de trasplantados renales). Nos fueron devueltas por correos cinco cartas debido a dirección incompleta, persona desconocida o traslado de domicilio y recibimos de los trasplantados 106 cartas de las que anulamos seis, bien porque los cuestionarios no estaban contestados o porque lo estaban de forma incorrecta.

Esto significa que el porcentaje total de pacientes trasplantados que participaron en esta investigación fue de $43,63 \%$, cuya distribución en función del órgano trasplantado fue: riñón $(38,65 \%)$, hígado $(45,45 \%)$ y corazón $(72 \%)$.

\section{Material testológico}

A continuación se hace una descripción de los instrumentos psicológicos utilizados en este estudio:

Encuesta psicosocial: fue elaborada por los autores del presente trabajo y evaluaba áreas muy diversas: datos sociodemográficos (sexo, edad, estado civil, nivel de estudios, etc.), datos relacionados con el trasplante de órganos (órgano trasplantado, fecha del trasplante, hospitalización tras el trasplante, reingresos a consecuencia del trasplante, etc.), datos relacionados con los cuidados e información médica (valoración de las actuaciones médicas e intervención quirúrgica y de la información sobre el proceso de trasplante), datos relacionados con la adherencia al tratamiento (seguimiento de las pautas de tratamiento y asistencia a las revisiones médicas) y otros datos (relaciones familiares, expectativas hacia la enfermedad, etc.).

Cuestionario de ansiedad estado-rasgo (Spielberger, Gorsuch y Lushene, 1982): contiene dos escalas, cada una con 20 items, que miden la ansiedad estado y la ansiedad rasgo.

La ansiedad estado hace referencia a cómo se siente el sujeto en el momento actual, es decir, evalúa un estado o condición emocional transitorio del organismo humano que se caracteriza por sentimientos subjetivos, conscientemente percibidos, de tensión y aprensión, así como una hiperactividad del sistema nervioso autonómico. Esta ansiedad puede variar en el tiempo y fluctuar en intensidad. Los items de esta escala tienen cuatro alternativas de respuestas: nada (0), algo (1), bastante (2) y mucho (3).

La ansiedad rasgo evalúa cómo se siente el sujeto habitualmente, es decir, señala una estable propensión ansiosa por la que difieren los sujetos en su tendencia a percibir las situaciones como amenazadoras y a elevar, consecuentemente, su ansiedad estado. Cada ítem de esta escala contiene cuatro alternativas de respuestas: casi nunca (0), a veces (1), a menudo (2) y casi siempre (3).

El rango de puntuaciones de cada una de las escalas de este cuestionario oscila entre 0 (ausencia de ansiedad) y 60 (máxima ansiedad).

Inventario de depresión de Beck (Beck, Rush, Shaw y Emery, 1979): está constituido por 21 items que intentan detectar y cuantificar el síndrome depresivo: emociones negativas, nivel de actividad, problemas de interacción, sentimientos de menosprecio, inadecuación y culpa, y síntomas físicos. Cada ítem consta de cuatro alternativas de respuestas (excepto el ftem número seis que tiene cinco alternativas) de las cuales el sujeto ha de elegir una. Se puntúa según una escala de 0 a 3 puntos y el rango de puntuaciones del inventario oscila entre 0 (ausen- 
cia de depresión) y 63 (depresión grave). Listado de pensamientos (Beck, Brown, Eidelson, Steer y Riskind, 1987): contiene 26 items agrupados en cinco situaciones y de ellos 12 se refieren a pensamientos automáticos ansiosos y 14 a depresivos. Cada ítem se contesta según una escala de cinco puntos: nunca (0), raramente (1), algunas veces (2), frecuentemente (3) y casi siempre (4). El rango de puntuaciones oscila entre 0 y 48 para la escala de pensamientos ansiosos y entre 0 y 56 para la escala de pensamientos depresivos. Mientras más alta sea la puntuación mayor es la presencia de dichos pensamientos.

Escala de autoconcepto (Garanto, 1984): es una escala multidimensional constituida por 100 items pero para el presente estudio sólo empleamos los 18 items que hacen referencia al autoconcepto físico. Estos se distribuyen en tres áreas, cada una con 6 items, que evalúan el autoconcepto, la autoestima y el autocomportamiento, es decir, cómo se percibe, siente y comporta el sujeto respecto a su propio cuerpo material, el estado de salud, el aspecto físico, su capacidad y su sexualidad. Cada ítem se contesta según una escala de cinco puntos: completamente falso (1), mayormente falso (2), parcialmente falso y parcialmente verdadero (3), mayormente verdadero (4) y completamente verdadero (5). El rango de puntuaciones para cada área oscila entre 6 y 30 puntos y mientras más alta sea la puntuación obtenida más satisfactorio es el autoconcepto del sujeto.

Cuestionario de conductas de afrontamiento (Muñoz, 1988): consta de 24 items que describen una situación problemática y una conducta ante esa situación. Estos items se agrupan en tres escalas, cada una con 8 items, que evalúan el tipo de conducta que utiliza el sujeto para hacer frente a las situaciones de estrés: enfrentamiento directo, escape-evitación y pasividad. Cada ítem tiene cinco alter- nativas de respuestas: nunca lo hago (0), casi nunca lo hago (1), a veces lo hago (2), casi siempre lo hago (3) y siempre lo hago (4). El rango de puntuaciones para cada una de las escalas oscila entre 0 y 32 puntos. Mientras más alta sea la puntuación obtenida por el sujeto, más característico es el uso de ese tipo de conducta concreto ante situaciones de estrés.

\section{RESULTADOS}

\section{Variables médicas}

Tipo de órgano trasplantado: tras comprobar que no existían diferencias estadísticamente significativas entre los tres grupos de trasplantados en una serie de variables sociodemográficas (sexo, edad, etc.), aplicamos la prueba de KolmogorovSmirnov en cada uno de los grupos en todas las variables psicológicas. Tras analizar los resultados, detectamos que excepto la variable "pensamientos depresivos» del grupo de renales, el resto de las variables seguían una distribución paramétrica. Consecuentemente, menos en esa variable que aplicamos el estadístico $\mathrm{K}$ de Kruskal-Wallis, en las demás utilizamos el AVAR paramétrico o análisis de varianza con la finalidad de analizar las diferencias psicológicas entre los tres grupos de trasplantados: renales, hepáticos y cardíacos. En la Tabla 2 se exponen los resultados obtenidos. Como puede apreciarse, no se hallaron diferencias entre los tres grupos en ninguna de las variables analizadas.

Hospitalización: para analizar la influencia que podía ejercer el tiempo que el paciente permanece hospitalizado tras el implante, seguimos los siguientes pasos: (1) tras aplicar la prueba de Kolmogorov-Smirnov, detectamos que esta variable no seguía una distribución paramétrica en los grupos de trasplantados renales y hepáticos, (2) consecuentemen- 
Tabla 2. Diferencias psicológicas entre los trasplantados renales, hepáticos y cardíacos

\begin{tabular}{lcccc}
\hline & \multicolumn{3}{c}{ Puntuaciones medias (+) } & \\
\cline { 2 - 4 } $\begin{array}{l}\text { Variables } \\
\text { psicológicas }\end{array}$ & $\begin{array}{c}\text { Trasplantados } \\
\text { renales }\end{array}$ & $\begin{array}{c}\text { Trasplantados } \\
\text { hepáticos }\end{array}$ & $\begin{array}{c}\text { Trasplantados } \\
\text { cardíacos }\end{array}$ & Significación \\
\hline - Ansiedad estado & 20,35 & 16,59 & 19,00 & 0,577 \\
- Ansiedad rasgo & 22,12 & 23,70 & 19,40 & 0,551 \\
- Depresión & 7,31 & 8,75 & 10,94 & 0,278 \\
- Pensamientos depresivos & $\mathbf{9 , 5 7}$ & 6,80 & $\mathbf{9 , 3 9}$ & $\mathbf{0 , 6 1 1}$ \\
- Pensamientos ansiosos & 7,30 & 7,37 & 7,00 & 0,984 \\
- Autoconcepto físico & 21,51 & 23,68 & 23,41 & 0,056 \\
- Autoestima física & 22,04 & 22,33 & 22,71 & 0,851 \\
- Autocomportamiento físico & 21,47 & 22,14 & 22,28 & 0,699 \\
- Afrontamiento directo & 13,84 & 14,00 & 16,81 & 0,154 \\
- Pasividad & 16,09 & 14,45 & 14,81 & 0,354 \\
- Escape-evitación & 14,14 & 14,05 & 15,47 & 0,675 \\
\hline
\end{tabular}

Nota: (+) medias procedentes de las puntuaciones directas.

te, aplicamos el estadístico K de KruskalWallis y comprobamos que las diferencias entre los grupos de trasplantados era significativa, (3) para saber entre qué grupos se hallaban las diferencias aplicamos el estadístico U de Mann-Whitney y detectamos que el tiempo medio que los trasplantados renales permanecían hospitalizados tras el implante era significativamente menor (19,19 días) que los trasplantados hepáticos $(34,27$ días $)$ y que los trasplantados cardíacos $(32,25$ días), no existiendo diferencias entre estos dos últimos grupos, (4) en cada uno de los grupos de trasplantados, hicimos dos subgrupos en función del mayor o menor tiempo de hospitalización tras el implante. Para ello seleccionamos en cada uno de los grupos el valor del percentil cincuenta: 14 días (trasplantados renales), 27 días (trasplantados hepáticos) y 20 días (trasplantados cardíacos), (5) en los dos subgrupos (más y menos tiempo de hospitalización) de trasplantados renales aplicamos la prueba de Kolmogorov-Smirnov y comprobamos que todas las variables seguían una distribución paramétrica. No hubo necesidad de aplicar esta prueba en los subgrupos formados dentro de los grupos de hepáticos y cardíacos debido al menor número de sujetos, y (6) por último, aplicamos los estadísticos $\mathrm{T}$ de Student-Fisher en el grupo de renales y $U$ de Mann-Whitney en los grupos de hepáticos y cardíacos, todo ello con la finalidad de comparar las variables psicológicas en función de la variable tiempo de hospitalización. En la Tabla 3 se exponen los resultados obtenidos. Como puede apreciarse, en ninguno de los grupos de trasplantados se hallaron diferencias entre los subgrupos en función del tiempo que habían permanecido ingresados en el hospital tras la implantación del injerto.

Por otro lado, para analizar la influencia que podía ejercer el hecho de reingresar en el hospital debido a complicaciones médicas tras el trasplante, seguimos los siguientes pasos: (1) dado que es una variable cualitativa con dos niveles ( $s i ́$ reingreso / no reingreso) aplicamos la prueba estadística Chi-Cuadrado y detectamos que no había diferencias estadísticamente significativas entre los tres grupos de trasplantados (renales, hepáticos y cardíacos) en esta variable, (2) como el porcentaje de pacientes que había reingresado en cada uno de los grupos era mínimo $(30,50 \%$ de los trasplantados renales, $26,98 \%$ de los trasplantados hepáticos y $29,41 \%$ de los trasplantados cardíacos), aplicamos directamente el estadístico U de Mann-Whitney para 
Tabla 3. Diferencias psicológicas entre los trasplantados en función del tiempo que han permanecido hospitalizados tras la implantación del injerto

\begin{tabular}{|c|c|c|c|c|c|c|c|c|c|}
\hline \multirow[b]{2}{*}{$\begin{array}{l}\text { Variables } \\
\text { psicologicas }\end{array}$} & \multicolumn{3}{|c|}{$\begin{array}{c}\text { Trasplantados renales } \\
(+)\end{array}$} & \multicolumn{3}{|c|}{$\begin{array}{c}\text { Trasplantados hepáticos } \\
(++)\end{array}$} & \multicolumn{3}{|c|}{$\begin{array}{c}\text { Trasplantados cardíacos } \\
(++)\end{array}$} \\
\hline & $\begin{array}{l}<14 \\
\text { días }\end{array}$ & $\begin{array}{l}>14 \\
\text { días }\end{array}$ & Signif. & $\begin{array}{l}<27 \\
\text { días }\end{array}$ & $\begin{array}{l}>27 \\
\text { días }\end{array}$ & Signif. & $\begin{array}{l}<20> \\
\text { días }\end{array}$ & $\begin{array}{c}20 \\
\text { días }\end{array}$ & Signif. \\
\hline $\begin{array}{l}\text { - Ansiedad estado } \\
\text { - Ansiedad rasgo } \\
\text { - Depresión } \\
\text { - Pensamientos depresivos } \\
\text { - Pensamientos ansiosos } \\
\text { - Autoconcepto físico } \\
\text { - Autoestima física } \\
\text { - Autocomport. físico } \\
\text { - Afrontamiento directo } \\
\text { - Pasividad } \\
\text { - Escape-evitación }\end{array}$ & $\begin{array}{r}20,57 \\
22,38 \\
6,73 \\
9,62 \\
7,29 \\
21,46 \\
22,00 \\
20,64 \\
16,55 \\
12,85\end{array}$ & $\begin{array}{r}20,11 \\
21,84 \\
7,88 \\
9,52 \\
7,30 \\
21,55 \\
22,08 \\
22,40 \\
15,54 \\
15,58\end{array}$ & $\begin{array}{l}0,453 \\
0,152 \\
0,614 \\
0,223 \\
0,772 \\
0,171 \\
0,341 \\
0,290 \\
0,648 \\
0,222\end{array}$ & $\begin{array}{r}8,95 \\
9,19 \\
9,85 \\
8,78 \\
8,69 \\
12,09 \\
10,75 \\
12,50 \\
9,00 \\
9,50\end{array}$ & $\begin{array}{r}12,86 \\
10,59 \\
10,17 \\
11,10 \\
10,15 \\
9,80 \\
10,25 \\
8,06 \\
13,20 \\
11,50\end{array}$ & $\begin{array}{l}0,148 \\
0,591 \\
0,902 \\
0,375 \\
0,558 \\
0,396 \\
0,849 \\
0,092 \\
0,118 \\
0,448\end{array}$ & $\begin{array}{r}5,17 \\
7,40 \\
7,43 \\
9,13 \\
7,94 \\
10,14 \\
9,56 \\
10,13 \\
7,86 \\
5,67\end{array}$ & $\begin{array}{l}8,57 \\
6,75 \\
8,50 \\
7,88 \\
9,06 \\
6,13 \\
6,21 \\
6,88 \\
8,13 \\
8,88\end{array}$ & $\begin{array}{l}0,114 \\
0,769 \\
0,642 \\
0,598 \\
0,634 \\
0,080 \\
0,144 \\
0,170 \\
0,907 \\
0,144\end{array}$ \\
\hline
\end{tabular}

Nota: (+) medias procedentes de las puntuaciones directas, $(++)$ medias procedentes de los rangos. 3

comparar las variables psicologicas en función del factor reingreso dentro de cada uno de los grupos de trasplantados. En la Tabla 4 se exponen los resultados obtenidos. Como puede apreciarse sólo se hallaron diferencias significativas en el grupo de cardíacos, en el sentido de que los pacientes que habían sufrido un reingreso debido a alguna complicación médica tras la implantación del corazón mostraron, por un lado, más ansiedad estado ( $p<0,01)$, ansiedad rasgo $(p<0,01)$, depresión $(\mathrm{p}<0,01)$ y pensamientos ansiosos $(p<0,05) y$, por otro lado, peor autoconcepto físico $(\mathrm{p}<0,05)$.

\section{Variables psicosociales}

Tiempo transcurrido desde el implante: para analizar la influencia que podía ejercer el tiempo transcurrido desde que el paciente fue trasplantado, seguimos los siguientes pasos: (1) tras aplicar la prue-

Tabla 4. Diferencias psicológicas entre los trasplantados en función del factor reingreso en el hospital debido a alguna complicación médica

\begin{tabular}{|c|c|c|c|c|c|c|c|c|c|}
\hline \multirow[b]{2}{*}{$\begin{array}{l}\text { Variables } \\
\text { psicológicas }\end{array}$} & \multicolumn{3}{|c|}{$\begin{array}{c}\text { Trasplantados renales } \\
(++)\end{array}$} & \multicolumn{3}{|c|}{$\begin{array}{c}\text { Trasplantados hepáticos } \\
(++)\end{array}$} & \multicolumn{3}{|c|}{$\begin{array}{c}\text { Trasplantados cardíacos } \\
(++)\end{array}$} \\
\hline & $\begin{array}{c}\text { No } \\
\text { reingreso }\end{array}$ & $\begin{array}{c}\text { Sí } \\
\text { reingreso }\end{array}$ & Signif. & $\begin{array}{c}\text { No } \\
\text { reingreso }\end{array}$ & $\begin{array}{c}\text { Sf } \\
\text { reingreso }\end{array}$ & Signif. & $\begin{array}{c}\text { No } \\
\text { reingreso }\end{array}$ & $\begin{array}{c}\text { Sf } \\
\text { reingreso }\end{array}$ & Signif. \\
\hline - Ansiedad estado & 27,06 & 28,39 & 0,768 & 10,25 & 14,83 & 0,139 & 5,33 & 11,40 & 0,009 \\
\hline - An & 26,01 & 2750 & 0,728 & 10,00 & 12,00 & 0,512 & $\mathbf{5 , 5 5}$ & 12,38 & $0,005^{* *}$ \\
\hline - Depresión & 25,94 & 27,65 & 0,702 & 9,64 & 12,50 & 0,319 & 6,18 & 13,60 & $0,003^{* *}$ \\
\hline - Pensar & 24,87 & 22,31 & 0,543 & 9,2 & 13,50 & 0,134 & 7,8 & 11,80 & 0,138 \\
\hline - Pensamientos ansiosos & 25,70 & 25,12 & 0,893 & 8,89 & 13,10 & 0,146 & 7,08 & 11,60 & $0,014^{*}$ \\
\hline - Autoconcepto físico & 28,74 & 26,47 & 0,620 & 12,91 & 6,70 & 0,058 & 9,88 & 4,38 & $0,044^{\star}$ \\
\hline - Autoestima física & 25,87 & 26,26 & 0,928 & 11,75 & 8,60 & 0,318 & 9,46 & 5,63 & 0,159 \\
\hline - Autocomport. físico & 25,35 & 30,50 & 0,254 & 11,88 & 8,20 & 0,245 & 10,25 & 6,00 & 0,112 \\
\hline - Afrontamiento directo & 25,12 & 27,61 & 0,566 & 10,63 & 12,20 & 0,619 & 8,05 & 9,50 & 0,567 \\
\hline - Pasividad & 26,47 & 28,03 & 0,727 & 12,88 & 6,80 & 0,064 & 9,45 & 6,40 & 0,232 \\
\hline - Escape-evitación & 26,65 & 24,81 & 0,670 & 11,81 & 8,40 & 0,282 & 6,95 & 10,88 & 0,124 \\
\hline
\end{tabular}

Nota: $(++)$ medias procedentes de los rangos, $\left({ }^{\star}\right) \mathrm{p}<0,05,\left(^{\star \star}\right) \mathrm{p}<0,01$. 
ba de Kolmogorov-Smirnov, detectamos que esta variable seguía una distribución paramétrica en los tres grupos de trasplantados, (2) consecuentemente, realizamos un análisis de varianza y no detectamos diferencias significativas entre los grupos en esta variable, (3) dado que en los grupos de trasplantados hepáticos $\mathrm{y}$ cardíacos al hacer los subgrupos en función del tiempo transcurrido desde el implante (0-12 meses, 12-24 meses y >24 meses) había valores de la variable con muy pocos sujetos, sólo pudimos analizar la influencia de esta variable en los trasplantados renales donde aplicamos, en primer lugar, el estadístico K de KruskalWallis y, en segundo lugar, la U de MannWhitney para detectar entre qué grupos se hallaban las diferencias. En la Figura 1 aparecen las tres variables que resultaron significativas: pensamientos depresivos $(\mathrm{p}<0,05)$, pensamientos ansiosos $(\mathrm{p}<0,05)$ y autoestima física $(p<0,05)$. Todas ellas se comportan en forma de «U» (pensamientos ansiosos y depresivos en forma de «U normal» y autoestima física, dado que puntúa al revés, en forma de «U invertida»), es decir, los trasplantados renales tienen más pensamientos (ansio- sos y depresivos) y peor autoestima física durante el primer año (0-12 meses) y a partir de los dos años (>24 meses) de haber sido trasplantados, no existiendo diferencias significativas entre estos dos períodos pero sí cuando se comparan ambos con el intervalo 12-24 meses. Dada la importancia de estas tres variables en la recuperación psicológica de los trasplantados renales, llevamos a cabo un análisis de items mediante el estadístico U de Mann-Whitney para detectar cuáles eran aquellos que tenían más peso, todos ellos quedan reflejados en la Tabla 5 .

Expectativas hacia la enfermedad: dado que esta variable estaba constituida por cuatro niveles (1. «estoy seguro de que superaré la enfermedad y sus consecuencias y que rápidamente volveré a ser el de siempre», 2. "mi enfermedad me ha causado muchos problemas, pero creo que los superaré relativamente pronto y volveré a estar como antes», 3. "mi enfermedad me ha puesto al límite, tanto físicamente como mentalmente, pero estoy luchando para superarlo y creo que seguramente volveré a ser el de siempre», y 4. «me siento agotado y muy débil por mi

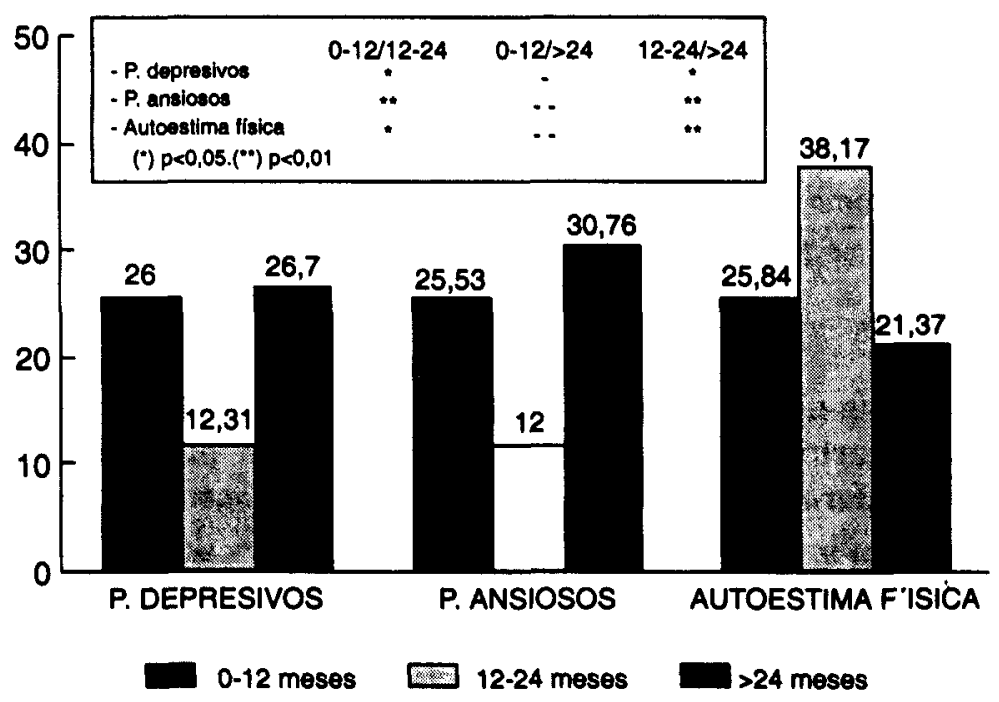

Figura 1. Evolución psicológica de los trasplantados renales 
Tabla 5. Diferencias psicológicas entre los trasplantados renales en función del tiempo transcurrido desde el implante. Análisis de items

Pensamientos depresivos (++)

Items $\quad 0.12$ meses $12-24$ meses Significación

- Cuando estoy en una reunión, pienso que siempre fracaso en mis relaciones sociales $16,039,94 \quad 0,039^{*}$

- Cuando estoy con un amigo pienso que la gente ya no me respeta

$15,75 \quad 10,50$

$0,035^{\star}$

- Ya no soy físicamente atractivo

16,25

9,50

$0,012^{\star}$

Items

12-24 meses >24 meses Significación

- Cuando estoy en una reunión, pienso que siempre fracaso en mis relaciones sociales

10,78

19,33

$0,015^{*}$

\begin{tabular}{|c|c|c|c|}
\hline \multicolumn{4}{|c|}{ Pensamientos ansiosos (++) } \\
\hline Items & $0-12$ meses & 12-24 meses & Significación \\
\hline $\begin{array}{l}\text { - Cuando siento dolor o malestar físico, pienso que voy a } \\
\text { sufrir una lesión }\end{array}$ & 16,25 & $\mathbf{9 , 5 0}$ & $0,012^{\star}$ \\
\hline Items & 12-24 meses & $>24$ meses & Significación \\
\hline $\begin{array}{l}\text { - Cuando siento dolor o malestar físico, pienso que debe } \\
\text { estarme ocurriendo algo que va a estropear mi aspecto físico }\end{array}$ & 11,00 & 19,25 & $0,010^{*}$ \\
\hline $\begin{array}{l}\text { - Cuando siento dolor o malestar físico, pienso que voy a } \\
\text { sufrir una lesión }\end{array}$ & 10,50 & 18,85 & $0,008^{* *}$ \\
\hline $\begin{array}{l}\text { - Cuando siento dolor o malestar físico pienso: ¿qué pasaría } \\
\text { si nadie llegase a tiempo de ayudarme? }\end{array}$ & 9,50 & 19,24 & $0,031^{*}$ \\
\hline $\begin{array}{l}\text { - Cuando siento dolor o malestar físico, pienso que tengo } \\
\text { alguna cosa muy mala }\end{array}$ & 10,00 & 19,63 & $0,004^{\star \star}$ \\
\hline - Voy a perder la cabeza & 11,50 & 19,06 & $0,024^{\star}$ \\
\hline _ Va a pasarle algo a un ser querido & 9,39 & 19,85 & $0,003^{* *}$ \\
\hline
\end{tabular}

\begin{tabular}{cccc} 
- Va a pasarle algo a un ser querido & 9,39 & 19,85 & $0,003^{\star *}$ \\
\hline Autoestima física $(++)$ & & & \\
\hline - No soy ni muy gordo ni muy delgado & $0-12$ meses & $12-24$ meses & Significación \\
\hline Items & 13,02 & 21,28 & $0,011^{*}$ \\
\hline - No soy ni muy gordo ni muy delgado & $12-24$ meses & $>24$ meses & Significación \\
\hline - Me gusta mi apariencia tal como es & 24,61 & 15,71 & $0,016^{\star}$ \\
\hline
\end{tabular}

Nota: A más puntuación se está más de acuerdo con la frase, $(++)$ medias procedentes de los rangos, $\left({ }^{*}\right) \mathrm{p}<0,05$, (**) $\mathrm{p}<0,01$.

enfermedad y hay veces que no sé si realmente seré capaz de superarlo»), no consideramos adecuado comparar las variables psicológicas en cada grupo de trasplantados, ya que metodológicamente no era correcto debido al escaso número de sujetos que había en algunos valores de esta variable en cada uno de los grupos. Por esta razón, analizamos los datos en su totalidad, es decir, sin hacer una distinción en función del órgano trasplantado. En primer lugar, aplicamos el estadístico $\mathrm{K}$ de Kruskal-Wallis para comparar las variables psicológicas en función de las diferentes expectativas que los trasplantados mostraban hacia su enfermedad y, en segundo lugar, en aquellas variables que resultaron significati- 
vas aplicamos el estadístico U de MannWhitney para saber entre qué grupos se hallaban las diferencias. En la Tabla 6 se exponen los resultados obtenidos. Como puede apreciarse, los trasplantados con unas expectativas más negativas hacia su enfermedad presentan, por un lado, más ansiedad estado $(p<0,01)$, ansiedad rasgo $(p<0,01)$, depresión $(p<0,01)$ y pensamientos depresivos $(p<0,01)$ y, por otro lado, peor autoconcepto físico $(p<0,01)$, autoestima física $(p<0,05)$ y autocomportamiento físico $(p<0,01)$.

Tipo de relaciones familiares tras el trasplante: esta variable estaba constituida por tres niveles (1. «las relaciones son mejores», 2. "las relaciones son las mismas que antes de ser trasplantado", y 3 . "las relaciones han empeorado»), pero como el número de trasplantados con peores relaciones tras el trasplante en cada uno de los grupos era mínimo, consideramos más adecuado, al igual que ocurría con la variable expectativas hacia la enfermedad, analizar los datos en su totalidad. En primer lugar, aplicamos el estadístico K de Kruskal-Wallis para comparar las variables psicológicas en función del tipo de relaciones familiares tras el trasplante y, en segundo lugar, en aquellas variables que resultaron significativas aplicamos el estadístico U de Mann-Whit-

Tabla 6. Diferencias psicológicas entre los trasplantados en función de las diferentes expectativas hacia su enfermedad.

\begin{tabular}{|c|c|c|c|c|c|c|c|c|c|c|c|}
\hline \multirow[b]{2}{*}{ Variables psicológicas } & \multicolumn{6}{|c|}{ Expectativas hacia la enfermedad (++) } & \multicolumn{4}{|c|}{ Contrastes } & \multirow[b]{2}{*}{ 3-4 } \\
\hline & (1) & (2) & (3) & (4) & Signif. & $1-2$ & $1-3$ & $1-4$ & $2-3$ & $2-4$ & \\
\hline - Ansiedad estado & 29,41 & 42,40 & 60,83 & 65,32 & $0,000^{\star \star}$ & * & ** & ** & * & ** & - \\
\hline - Ansiedad rasgo & 30,69 & 33,23 & 61,13 & 61,17 & 0,000 ** & - & ** & ** & ** & * * & - \\
\hline - Depresión & 31,53 & 38,52 & 62,14 & 58,18 & 0,000 ** & - & * & ** & ** & * & - \\
\hline - Pensamient. depresivos & 32,51 & 38,97 & 54,44 & 51,70 & $0,006^{* *}$ & - & «* & * & * & - & - \\
\hline - Pensamientos ansiosos & 31,49 & 40,78 & 48,53 & 53,72 & $0,142^{\star *}$ & - & - & - & - & - & - \\
\hline - Autoconcepto físico & 56,04 & 48,64 & 28,83 & 32,22 & $0,000^{* *}$ & - & ** & * & ** & - & - \\
\hline - Autoestima física & 48,03 & 49,27 & 26,85 & 37,50 & $0,013^{\star \star}$ & - & ** & - & ** & - & - \\
\hline - Autocomportam. físico & 53,76 & 48,66 & 29,80 & 31,40 & $0,002^{\star *}$ & - & ** & * & * * & - & - \\
\hline - Afrontamiento directo & 48,07 & 36,10 & 35,88 & 54,50 & $0,073^{\star *}$ & - & - & - & - & - & - \\
\hline — Pasividad & 39,47 & 50,20 & 46,55 & 49,86 & $0,388^{* *}$ & - & - & - & - & - & - \\
\hline - Escape-evitación & 41,89 & 43,55 & 43,05 & 45,94 & $0,975^{*}$ * & 一 & 一 & - & - & - & - \\
\hline
\end{tabular}

Nota: Las expectativas hacia la enfermedad tienen distinta graduación: desde (1) que son expectativas positivas a (4) que son expectativas negativas, (++) medias procedentes de los rangos, $(*) p<0,05,\left(^{*}\right) p<0,01$.

Tabla 7. Diferencias psicológicas entre los trasplantados en función del tipo de relaciones familiares tras el trasplante

\begin{tabular}{|c|c|c|c|c|c|c|c|}
\hline \multirow[b]{2}{*}{ Variables psicológicas } & \multicolumn{4}{|c|}{ Relaciones familiares (++) } & \multicolumn{3}{|c|}{ Contrastes } \\
\hline & Mejores (1) & Mismas (2) & Peores (3) & Signif. & $1-2$ & $1-3$ & $2-3$ \\
\hline - Ansiedad estado & 38,47 & 46,67 & 81,40 & $0,002^{\star *}$ & - & ** & ** \\
\hline - Ansiedad rasgo & 41,12 & 41,89 & 74,20 & $0,017^{*}$ & - & $\star$ & * \\
\hline - Depresión & 41,06 & 43,65 & 77,30 & $0,011^{\star}$ & - & * & * \\
\hline - Pensamientos depresivos & 41,49 & 39,88 & 73,00 & $0,014^{*}$ & - & - & * \\
\hline - Pensamientos ansiosos & 43,99 & 42,16 & $\mathbf{5 5 , 0 0}$ & 0,605 & - & - & - \\
\hline - Autoconcepto físico & 51,82 & 45,03 & 32,80 & 0,243 & - & - & - \\
\hline - Autoestima física & 47,49 & 44,84 & 20,90 & 0,092 & - & - & - \\
\hline - Autocomportamiento físico & $\mathbf{5 4 , 6 5}$ & 43,16 & 19,00 & $0,008 *$ & - & * & * \\
\hline - Afrontamiento directo & 49,70 & 39,09 & 66,40 & 0,025 * & * & - & * * \\
\hline — Pasividad & 44,60 & 46,42 & 53,25 & 0,811 & - & - & - \\
\hline - Escape-evitación & 49,05 & 40,67 & 44,00 & 0,333 & - & - & 一 \\
\hline
\end{tabular}

Nota: (++) medias procedentes de los rangos, $\left({ }^{*}\right) \mathrm{p}<0,05,\left(^{* *}\right) \mathrm{p}<0,01$. 
ney para saber entre qué grupos se hallaban las diferencias. En la Tabla 7 se exponen los resultados obtenidos. Como puede apreciarse, cuando las relaciones familiares tras el trasplante empeoran, los pacientes presentan por un lado, más ansiedad estado $(p<0,01)$, ansiedad rasgo $(p<0,05)$, depresión $(p<0,05)$, pensamientos depresivos $(p<0,05)$ y conductas de afrontamiento directo $(p<0,05) y$, por otro lado, peor autocomportamiento físico $(p<0,01)$.

\section{DISCUSIÓN}

A partir de los resultados obtenidos, identificamos la influencia diferencial que ejercen las variables analizadas en este estudio sobre la recuperación psicológica de los trasplantados:

\section{Variables médicas}

Tipo de órgano trasplantado: no se hallaron diferencias psicológicas entre los trasplantados renales, hepáticos y cardíacos. Esto nos lleva a pensar que en los tres grupos el impacto del trasplante es el mismo. En líneas generales, debido a que un rechazo renal no genera consecuencias tan dramáticas (el paciente puede volver a diálisis e incluso recibir otro riñón) como un rechazo hepático o cardíaco (el paciente puede morir), en principio, podríamos pensar que el primer grupo se halla mejor psicológicamente que los otros dos grupos y, sin embargo, los datos obtenidos apuntan que en los tres grupos de trasplantados la experiencia del trasplante es igual de estresante. En relación a esta variable, sólo hemos hallado un estudio que tras comparar varios grupos de trasplantados (pulmonares, hepáticos y cardíacos) concluye que los dos últimos tienen peor funcionamiento a nivel físico, psicológico y social (Littlefield, Abbey, Fiducia y Cardella, 1996).

Hospitalización: mientras que el tiempo de hospitalización tras el implante (menor en los renales que en los hepáticos y cardíacos) no ejerce una influencia significativa en la recuperación psicologica de los trasplantados, por el contrario, el reingreso en el hospital debido a alguna complicación médica tras el implante cardíaco, hace que estos pacientes se hallen peor psicológicamente. En esta línea, tendríamos que plantearnos la siguiente cuestión: ¿Por qué no ocurre lo mismo en los trasplantados renales y en los hepáticos? Una posible respuesta es que el corazón puede tener un significado especial para los sujetos: «es el centro de la vida», "es el motor principal del organismo", "es el que sufre y padece todo tipo de emociones", etc. Ello implica que ante una recaída y posible pérdida del injerto, los pacientes muestren ansiedad no sólo por la posibilidad de morir, sino también porque ven amenazadas las funciones (médicas y psicológicas) que ellos atribuyen al corazón, de ahí que tengan más reacciones ansiosas y depresivas que, sin duda, contribuyen a que tengan una percepción más negativa respecto a su propio cuerpo material, aspecto físico, estado de salud, etc.

Por otro lado, hemos de tener en cuenta, que estos desequilibrios psicológicos que presentan los trasplantados cardíacos que han sufrido un reingreso pueden tener un efecto muy negativo en la recuperación física del paciente. En este sentido, algunos estudios han demostrado que los problemas psiquiátricos tras el trasplante, no sólo aumentan el riesgo de padecer una infección, sino también la probabilidad de incumplir las prescripciones terapéuticas (Deschields, McDonough, Mannen y Miller, 1996; París, Muchmore, Pribil, Zuhdi y Cooper, 1994). Además, los pacientes suelen tener sentimientos ambiguos hacia el 
equipo de trasplantes, por un lado, agradecimiento por haberles salvado la vida pero, por otro lado, agresividad por la dependencia de ellos y porque descubren que el trasplante no es volver a nacer con un cuerpo sano, sino que siguen siendo pacientes que necesitan cuidados y vigilancia médica (Mai, Kenzie y Kostuk, 1990).

\section{Variables psicosociales}

Tiempo transcurrido desde el implante: esta variable fue analizada en los trasplantados renales y comprobamos que durante el primer año (0-12 meses) y a partir de los dos años (>24 meses) de haber recibido el implante, estos pacientes se hallaban peor psicológicamente, es decir, presentaban más pensamientos (ansiosos y depresivos) y peor autoestima física. Estos resultados son semejantes a los hallados en otros estudios, así por ejemplo, Jones et al. (1988) concluyen en una muestra constituida por 38 trasplantados cardíacos, por un lado, que el $19 \%$ de los pacientes presentaban más ansiedad a los cuatro meses de seguimiento que tras el alta hospitalaria y, por otro lado, que el $43 \%$ presentaba más ansiedad a los doce meses que a los ocho meses después del trasplante. Así mismo, Moore, Burrows y Hardy (1997) encuentran en un grupo de 32 trasplantados hepáticos que a medida que pasa el tiempo pueden surgir episodios de ansiedad, los cuales dependen del tipo de apoyo recibido. Llegados a este punto, tendríamos que plantearnos la siguiente cuestión: ¿Por qué en la muestra estudiada los desequilibrios psicológicos son más patentes al principio (0-12 meses) y al final (>24 meses) de los períodos comparados tras la implantación del injerto?. Una posible explicación es que los trasplantados renales atraviesan por tres fases tras el implante:
- Fase de alerta: una vez superada la hospitalización, durante el primer año (0-12 meses) los pacientes, por un lado, han de mantener unas pautas de tratamiento muy rígidas (alimentación, medicación, ejercicio físico, etc.) y, por otro lado, han de integrarse en un entorno sociolaboral que muchas veces no es el más adecuado por sus condiciones físicas. A todo esto hay que añadir, por un lado, que al principio los pacientes temen la posibilidad de poder rechazar el órgano ante la más mínima sintomatología, es decir, se hallan en una situación de constante alerta y, por otro lado, que la dinámica familiar puede encontrarse alterada por las consecuencias del trasplante, especialmente por haber soportado la angustiante espera del órgano. Todo ello repercute negativamente en la recuperación psicológica de los pacientes trasplantados.

- Fase de adaptación: a partir del primer año (12-24 meses) de haber recibido el implante renal, la mayoría de los pacientes se han habituado a las prescripciones terapéuticas, se han integrado en el entorno sociolaboral, dejan de temer el rechazo del órgano ya que aprecian que su evolución física es favorable y, además, la familia está más recuperada psicológicamente por lo que puede prestarle más apoyo. Todo ello hace que los trasplantados renales se adapten a todas las circunstancias que conlleva el trasplante $y$, consecuentemente, que disminuyan los desequilibrios psicológicos durante esta fase.

- Fase de agotamiento: a partir de los dos años (>24 meses) del implante renal de nuevo aumentan los desequilibrios psicológicos. Una posible explicación sería, por un lado, que vuelve a aparecer el temor a la pérdida del injerto, ya que la mayoría de los pacientes saben que a medida que pasa el tiempo disminuye la supervivencia del injerto y, por otro lado, que los pacientes están cansados y agota- 
dos de su situación, es decir, aparecen desilusiones ante unas expectativas frustradas pues descubren que el trasplante no significa una vida como la de antes de empezar la enfermedad, en definitiva, el trasplante ofrece la oportunidad de seguir viviendo pero siempre bajo vigilancia médica.

Todo ello conlleva que durante el primer año (0-12 meses) y a partir de los dos años (>24 meses) del trasplante, los pacientes muestren pensamientos depresivos (por ejemplo, «soy un fracaso en mis relaciones sociales», «la gente ya no me respeta», "no soy físicamente atractivo») y ansiosos (por ejemplo, "voy a sufrir una lesión», "me debe estar ocurriendo algo que va a estropear mi aspecto físico", "¿qué pasaría si nadie llegase a tiempo de ayudarme si siento dolor o malestar físico?», "tengo alguna cosa muy mala», "voy a perder la cabeza», "va a pasarle algo a un ser querido"). Estos pensamientos implican, sobre todo, una visión negativa de uno mismo e ideas catastrofistas sobre la propia salud que repercuten negativamente en la autoestima física de los trasplantados, es decir, en sus sentimientos hacia su salud, su aspecto físico (por ejemplo, «no les gusta su apariencia tal como es»), etc.

Expectativas hacia la enfermedad: los trasplantados con unas expectativas más negativas hacia su enfermedad presentan más trastornos psicológicos. En este sentido, los pacientes que consideran que su enfermedad les ha puesto al límite, tanto física como mentalmente (aunque siguen luchando para superarla) y los que se sienten agotados y débiles por su enfermedad (dudando si lograrán superarla) son los que muestran más ansiedad (estado y rasgo) y conductas y pensamientos depresivos. Esto es lógico, ya que al tener unas expectativas negativas es más probable que tengan pensamientos irracionales sobre el funcionamiento del órgano implantado, esto les lleva a pensar en la posibilidad de una evolución desfavorable del trasplante con temor al rechazo del órgano y, consecuentemente, a la muerte. Además, Leedham, Meyerowitz y Muirhead (1995), encontraron que a medida que los trasplantados tenían unas expectativas más negativas hacia su enfermedad, no sólo se adherían peor al tratamiento médico, sino que además disminuía su calidad de vida. Todo ello influye negativamente en el autoconcepto físico de los pacientes, es decir, en cómo se perciben (autoconcepto físico), sienten (autoestima física) y se compor$\tan$ (autocomportamiento físico) con respecto a su salud, su cuerpo, su aspecto físico, su capacidad y su sexualidad.

Por otro lado, hemos de tener en cuenta que otros estudios han demostrado que las expectativas que tienen los pacientes antes de la operación pueden ejercer una gran influencia, si éstas son demasiado altas (por ejemplo, no esperan tener ninguna complicación física ni psicológica) es más probable que se depriman (Dubovsky y Penn, 1980).

Tipo de relaciones familiares tras el trasplante: el hecho de que en algunos casos las relaciones familiares sean peores a raíz del trasplante, podría deberse al agotamiento psicológico de la familia originado por todas las tensiones y temores (por ejemplo, al rechazo del injerto y a la muerte) que han soportado durante la enfermedad y la espera del trasplante (Iruela et al., 1995). De hecho, en un reciente estudio donde se analizan las relaciones de pareja de 36 trasplantados cardíacos antes y después del implante, se concluye la presencia de un alto nivel de estrés en ambos miembros de la pareja (Konstam et al., 1998). En nuestro estudio, hallamos que cuando las relaciones familiares empeoran a raíz del trasplante suelen aumentar los trastornos psicológicos de los trasplantados, es decir, estos muestran más ansiedad (estado y rasgo) 
y más conductas y pensamientos depresivos. Ello parece lógico, ya que estos familiares debido a sus malas relaciones no se hallan capacitados para proveer apoyo físico y psicológico a los pacientes, lo que puede tener serias implicaciones en el bienestar de los mismos (Littlefield, 1992). En este sentido, uno de los predictores con más peso en el abandono del tratamiento es la falta de apoyo social y familiar (Kiley, Lam y Pollak, 1993). Las consecuencias de esta ausencia de apoyo son, por un lado, un aumento de los trastornos psicológicos en los trasplantados $y$, por otro lado, que éstos tengan un comportamiento inadecuado respecto a los aspectos físicos de su cuerpo (autocomportamiento físico): no cuidarse físicamente, no ser cuidadosos con su apariencia, dormir con dificultad, etc. Además, al no tener el apoyo que necesitan, muchos de estos pacientes intentan afrontar directamente los problemas, es decir, ante cualquier percance suelen llevar a cabo una serie de acciones directas y en cierto grado agresivas para alterar la situación (afrontamiento directo).

En resumen, podemos decir que aunque el tipo de órgano trasplantado y el tiempo de hospitalización no ejercen una influencia importante en la recuperación psicológica de los trasplantados, por el contrario, el reingreso en el hospital debido a alguna complicación médica, el tiempo transcurrido desde la fecha del implante, las expectativas hacia la enfermedad y las relaciones familiares tras el trasplante son variables que hemos de tener en cuenta en la evolución psicológica de los trasplantados.

Para finalizar, consideramos que la intervención de los psicólogos en este ámbito es fundamental para lograr los siguientes objetivos:

- Eliminar los desequilibrios psicologicos de los trasplantados y sus familia- res (Abbey y Farrow, 1998; Klapheke, 1999; Zipfel et al., 1998). Diversas técnicas derivadas de la Modificación de Conducta pueden ayudarnos en este propósito: relajación, reestructuración cognitiva, exposición, entrenamiento en habilidades sociales, etc. (Pérez, Garrido y Blanco, 1999). Los programas terapéuticos, no sólo han de implicar al paciente, sino también a sus familiares más allegados $\mathrm{y}$, además, han de tener en cuenta variables como las expectativas del paciente, circunstancias por las que atraviesa éste en el momento en el que se realiza la intervención, etc.

- Evaluar los candidatos a trasplante para seleccionar los más idóneos e intentar detectar futuras complicaciones (Menchon et al., 1997).

- Potenciar la donación de órganos, ya que el trasplante de órganos es una alternativa imposible si no existen donaciones. En este sentido, las causas de la escasez de órganos pueden provenir tanto de los familiares de pacientes diagnosticados de muerte cerebral (Blanco, Pérez, Murillo, Domínguez y Núñez, 1994; Domínguez, Murillo, Muñoz y Pérez, 1992; Pérez, Blanco, Murillo, Domínguez y Núñez, 1992; Pérez et al., 1992) como del propio personal sanitario (Pérez, Domínguez, Murillo y Núñez, 1993; Pérez, Martín, Navarro, Ruano y Blanco, 1995).

En definitiva, si los psicólogos intervenimos en ambas áreas (trasplante y donación de órganos), no sólo aumentaríamos el número de donaciones y por tanto de trasplantes, sino que además, por un lado, estaríamos haciendo algo positivo con los familiares de los donantes (por ejemplo, dar un significado a una muerte que es incomprensible) $y$, por otro lado, aumentaríamos la cantidad y calidad de vida de los trasplantados y lograríamos un mayor equilibrio en sus relaciones familiares. 


\section{REFERENCIAS BIBLIOGRÁFICAS}

Abbey, S., y Farrow, S. (1998). Group therapy and organ transplantation. International Journal of Group Psychotherapy, 48, 163-185.

Aranzábal, J., y Magaz, A. (1993). El trasplante renal. Beneficios clínicos y sociales. Revista Española de Trasplantes, 1, 43-55.

Beck, A.T., Brown, G., Eidelson, J.I., Steer, R.A., y Riskind, J.H. (1987). Differentiating anxiety and depression: $A$ test of the cognitive content-specificity hypothesis. Journal of Abnormal Psychology, 96, 179-183.

Beck, A.T., Rush, A.J., Shaw, B.F., y Emery, G. (1979). Cognitive therapy of depression. New York: Guilford Press.

Blanco, A., Pérez, M.A., Murillo, F., Domínguez, J.M., y Núñez, A. (1994). Análisis de actitudes y creencias que dificultan la donación de órganos y propuesta de un programa de intervención. Psicología Conductual, 2, 215-234.

Deschields, T.L., McDonough, E.M., Mannen, R.K., y Miller, L.W. (1996). Psychological and cognitive status before and after heart transplantation. Journal of Higher Education, 18, 62-69.

Dew, M.A., Roth, L.H., Schulberg, H.C., y Simmons, R.G. (1996). Prevalence and predictors of depression and anxiety related disorders during the year after heart transplantation. Journal of Higher Education, 18, 48-61.

Domínguez, J.M., Murillo, F., Muñoz, A., y Pérez, M.A. (1992). Psychological aspects leading to refusal of organ donation in southwest Spain. Transplantation Proceedings, 24, 25-26.

Dubovsky, S.L., y Penn, I. (1980). Psychiatric considerations in renal transplant surgery. Psychosomatics, 21, 481-491.

Evans, R.W. (1985). The quality of life of patients with end-stage renal disease. The New England Journal of Medicine, 312, 553-559.
Garanto, J. (1984). Las actitudes hacia sí mismo y su medición. Barcelona: Publicaciones y Ediciones de la Universidad de Barcelona.

Gudex, C.M. (1995). Health related quality of life in endstage renal failure. Quality of Life Research and International Journal of Quality of Life Aspects of Treatment, Care and Rehabilitation, 4, 359-366.

House, R.M., y Thompson, T.L. (1988). Psychiatric aspects of organ transplantation. Journal of the American Medical Association, 260, 535-539.

Iruela, L.M., Merino, J., y Lombardía, C. (1995). Psiquiatría de enlace en los enfermos trasplantados. Monografías de Psiquiatría, 3, 36-39.

Jones, B.M., Chang, V.P., Esmore, D., Spratt, P., Shanahan, M.X., Farnsworth, A.E., Keogh, A., y Downs, K. (1988). Psychological adjustment after cardiac transplantation. The Medical Journal of Australia, 149, 118-122.

Kiley, D.J., Lam, C.S., y Pollak, R. (1993). A study of treatment compliance following kidney transplantation. Transplantation, 55, 51-56.

Klapheke, M.M. (1999). The role of the psychiatrist in organ transplantation. Bulletin of the Menninger-Clinic, 63, 13-39.

Konstam, V., Surman, O., Hizzazi, K.H., Fierstein, J., Konstam, M., Turbett, A., Dec, G.W., Keck, S., Mudge, G., Flavell, C., McCormack, M., y Hurley, L. (1998). Marital adjustment in heart transplantation patients and their spouses: A longitudinal perspective. American Journal of Family Therapy, 26, 147-158.

Leedham, B., Meyerowitz, B.E., y Muirhead, J. (1995). Positive expectations predict health after heart transplantation. Health Psychology, 14, 74-79.

Littlefield, C. (1992). Social support and organ transplantation. En J. Craven y G.M. Rodin (Eds.), Psychiatric aspects of organ transplantation (pp. 51-65). Oxford: Oxford University Press. 
Littlefield, C., Abbey, S., Fiducia, D., y Cardella, C. (1996). Quality of life following transplantation of the heart, liver and lungs. Journal of Higher Education, 18, 36-47.

López, O.L., Estol, C., y Colina, I. (1992). Neurological complications after liver retransplantation. Hepatology, 16, 162166.

Mai, F.M., Kenzie, F.N., y Kostuk, W.J. (1990). Psychosocial adjustment and quality of life following heart transplantation. Canadian Journal of Psychiatry, 142, 527-537.

Menchón, J.M., González, A., Aymamí, N., Barjau, R., y Serrano, F. (1997). El trasplante. En J.E. Rojo y E. Cirera (Dirs.), Interconsulta psiquiátrica (pp. 501-511). Barcelona: Masson, S.A.

Moore, K.A., Burrows, G.D., y Hardy, K.J. (1997). Anxiety in chronic liver disease: Changes post transplantation. Stress Medicine, 13, 49-58.

Muñoz, M. (1988). Características de enfrentamiento: Aspectos perceptivos y motores. Tesis Doctoral, Universidad Complutense de Madrid.

París, W., Muchmore, J., Pribil, A., Zuhdi, N., y Cooper, D.K. (1994). Study of relative indices of psychosocial factors before and after heart transplantation and the influence of postransplantation psychosocial factors on heart transplantation outcome. Journal of Heart and Lung Transplantation, 13, 424-430.

Pérez, M.A., Blanco, A., Murillo, F., Domínguez, J.M., y Núñez, A. (1992). Psychological profile of families of severely traumatized patients. Relationship to organ donation for transplantation. Transplantation Proceedings, 24, 27-28.

Pérez, M.A., Blanco, A., Murillo, F., Domínguez, J.M., Sánchez, B., y Núñez, A. (1992). Psychological problems in the family members of gravely traumatised patients admitted into an intensive care unit. Intensive Care Medicine, 18, 278-281.
Pérez, M.A., Domínguez, J.M., Murillo, F., y Núñez, A. (1993). Actitudes del personal sanitario hacia la donación de órganos. Revista de Psicología de la Salud, 5, 3-18.

Pérez, M.A., Garrido, M., y Blanco, A. (1999). Impacto psicológico individual y familiar del trasplante. Aportaciones desde la Terapia de Conducta y la Terapia Familiar Sistémica. Cuadernos de Medicina Psicosomática, 49-50, 8-18.

Pérez, M.A., Martín, A., Navarro, A., Ruano, J.J., y Blanco, A. (1995). Programa de intervención psicológica en el personal de enfermería para potenciar la donación de órganos. Clínica y Salud, 6, 189-202.

Plutchik, L., Snyder, S., Drooker, M., Chodoff, L., y Sheiner, P. (1998). Methylphenidate in post liver transplant patients. Psychosomatics, 39, 118-123.

Simmons, R.G., Kamstra, L., y Thompson, C.R. (1981). Psychosocial adjustment five to nine years post-transplant. Transplantation Proceedings, 13, 40-43. Spielberger, C.D., Gorsuch, R.L., y Lushene, R.E. (1982). Cuestionario de ansiedad estado-rasgo. Madrid: TEA Ediciones, S.A.

Stukas, A.A., Dew, M.A., Switzer, G.E., DiMartini, A., Kormos, R.L., y Griffith, B.P. (1999). PTSD in heart transplant recipients and their primary family caregivers. Psychosomatics, 40, 212-221.

Tabler, J.B., y Frieson, R.L. (1990). Sexual concerns after heart transplantation. Journal of Heart Transplantation, 9, 397-403.

Van Der Velde, C.D. (1985). Body image of one's self and others: Developmental and clinical significance. American Journal of Psychiatry, 142, 527-537.

Zipfel, S., Loewe, B., Paschke, T., Immel, B., Lange, R., Zimmerman, R., Herzog, W., y Bergmann, G. (1998). Psychological distress in patients awaiting heart transplantation. Journal of Psychosomatic Research, 45, 465-470. 